\title{
Striving for an effective but parsimonious use of sedation in pediatric intensive care
}

\author{
Nienke J. Vet ${ }^{*}$, Saskia N. de Wildt, Dick Tibboel and Matthijs de Hoog
}

(c) 2016 Springer-Verlag Berlin Heidelberg and ESICM

\section{Dear Editor,}

We greatly appreciate the interest shown in our paper by Zimmerman and colleagues [1].

First of all, we acknowledge that the secondary mortality finding might have received more emphasis than warranted by the results of extensive causality analysis. Indeed, we also believe that it is highly unlikely that there is a relationship between the causes of death and daily sedation interruption (DSI) [2]. Nevertheless, as this finding was totally unexpected, it was reported in all openness in the conclusion of the study.

In answer to the questions raised, our sedation protocol includes a weaning strategy, in which the nurse can actively taper the sedation. COMFORT-B scores are performed at least every $8 \mathrm{~h}$ and if lower than targeted, nurses can decrease the morphine and midazolam stepwise. Morphine can be decreased by $10 \mathrm{mcg} / \mathrm{kg} / \mathrm{h}$ once a day and midazolam by $50 \mathrm{mcg} / \mathrm{kg} / \mathrm{h}$ every $8 \mathrm{~h}$. In addition to the assessment of the COMFORT-B score, the validated numeric rating scale (NRS), the Sophia Observation withdrawal Symptoms (SOS), and SOS-pediatric delirium (SOS-PD) scale are standardly used in our PICU [3]. Together, these scores help to differentiate between distress, pain, withdrawal, and/or delirium. In addition, before increasing sedatives, nurses evaluate if a non-pharmacological intervention is effective to reduce distress.

We acknowledge that in many PICUs morphine is used as the first-line analgo-sedative drug, with the potential

\footnotetext{
*Correspondence: n.vet@erasmusmc.nl

Intensive Care and Departments of Pediatrics and Pediatric Surgery, Erasmus MC - Sophia Children's Hospital, Dr. Molewaterplein 60, 3015 GJ Rotterdam, Netherlands
}

advantage of also providing analgesia. In our center, midazolam is used as the first-line sedative on the basis of the rationale that ICU treatment in itself is not so painful to warrant an opioid. Only when sedation is insufficient with midazolam will morphine be added, or when scores or clinical situation suggests pain. We are well aware of the risk of development of withdrawal and delirium after use of benzodiazepines, but data support that this is similarly prevalent with opioids [4].

Further, although we did not explicitly study the immune competency status, we have no reason to believe that there would be a difference between both groups. Diagnoses were similar between both groups and most patients $(67 \%)$ were admitted for a respiratory disorder, mainly a viral pneumonia. Reintubations were more frequent in the control group. Although the study was not completely blinded, to our own surprise, nurses and doctors found the study protocol quite complicated and only seldom concluded study group allocation from the infusion restart rate. Therefore, we believe that the risk of bias was minimized.

We agree that there is an increasing body of evidence for "less is more" in critical care, which the results of our study do not contradict. In lieu of the obvious unwanted effects of sedation, striving for an effective but parsimonious use of sedation in pediatric intensive care is imperative. This should be the objective of future, well-designed multicenter randomized controlled trials (RCT). A potential future RCT could compare continuous 'light sedation' with 'normal/conscious' sedation. This may result in less variation in sedation levels with less extra boluses throughout the day, as compared to DSI, and a reduced cumulative need for sedative drug doses in the 'light sedation' group.

\section{国


Accepted: 15 March 2016

Published online: 31 March 2016

\section{References}

1. Zimmerman JJ, Watson RS, Ely EW (2016) Daily sedation interruption in children warrants further study. Intensive Care Med. doi:10.1007/ s00134-016-4290-y

2. Vet NJ, de Wildt SN, Verlaat CWM, Knibbe CAJ, Mooij MG, van Woense JBM, van Rosmalen J, Tibboel D, de Hoog M (2016) A randomized controlled trial of daily sedation interruption in critically ill children. Intensive Care Med 42:233-244. doi:10.1007/s00134-015-4136-z
3. Ista E, de Hoog M, Tibboel D, Duivenvoorden HJ, van Dijk M (2013) Psychometric evaluation of the Sophia observation withdrawal symptoms scale in critically ill children. Pediatr Crit Care Med 14:761-769

4. Anand KJ, Willson DF, Berger J, Harrison R, Meert KL, Zimmerman J,

Carcillo J, Newth CJL, Prodhan P, Dean JM, Nicholson C, Eunice Kennedy Shriver National Institute of Child Health and Human Development Collaborative Pediatric Critical Care Research Network (2010) Tolerance and withdrawal from prolonged opioid use in critically ill children. Pediatrics 125:e1208-e1225 